\title{
Projective Synchronization of Hyperchaotic Financial Systems
}

\author{
Jiming Zheng and Bingfang Du \\ School of Science, Chongqing University of Posts and Telecommunications, Chongqing 400065, China \\ Correspondence should be addressed to Jiming Zheng; zhengjm0903@qq.com
}

Received 18 July 2014; Revised 26 October 2014; Accepted 1 November 2014

Academic Editor: Seenith Sivasundaram

Copyright (C) 2015 J. Zheng and B. Du. This is an open access article distributed under the Creative Commons Attribution License, which permits unrestricted use, distribution, and reproduction in any medium, provided the original work is properly cited.

Based on a special matrix structure, the projective synchronization control laws of the hyperchaotic financial systems are proposed in this paper. Put a hyperchaotic financial system as the drive system, via transformation of the system state variables, construct its response system, and then design the controller based on the special matrix structure. The given scheme is applied to achieve projective synchronization of the different hyperchaotic financial systems. Numerical experiments demonstrate the effectiveness of the method.

\section{Introduction}

Chaos in the economy was first discovered since 1985; it had made a tremendous impact on market economy. Chaos theory provides new approaches and ideas for financial crisis and other related issues. Synchronous development of the financial system is a real problem faced by many economists, such as how to achieve synchronous development in different countries and areas. In 1999, Mainieri and Rehacek [1] proposed a chaotic synchronous mapping; namely, driveresponse system can be synchronous with a desired scaling factor. In recent decades, projective synchronization $[2,3]$ of the chaotic financial system is also of concern. Scholars realized projective synchronization of three-dimensional fractional-order chaotic financial system and the integerorder one, respectively. Hyperchaotic system has two or more positive Lyapunov exponents. The economic system is a high-dimensional nonlinear system, whose chaos is mostly super chaos [4]. Achieving synchronization of hyperchaotic financial system is closer to the actual condition, so some researchers have investigated the synchronization of hyperchaotic financial system $[5,6]$. In this paper, based on a special matrix and conversion of state variables, we will realize projective synchronization of the identical and different hyperchaotic financial systems.

\section{Projective Synchronization of Hyperchaotic Financial Systems}

Consider the financial system [7] as follows:

$$
\begin{gathered}
\dot{x}=z+(y-a) x, \\
\dot{y}=1-b y-x^{2}, \\
\dot{z}=-x-c z,
\end{gathered}
$$

where $x, y$, and $z$ represent the interest rate, investment demand, and price index, respectively. The parameter $a$ is the saving, $b$ is the per-investment cost, and $c$ is the elasticity of demands of commercials. And they are positive constants.

Based on the chaotic finance of system (1), scholars found that the factors affecting the interest rate are related not only to investment demand and price index, but also to the average profit margin denoted by $w$. Therefore, the following improved chaotic finance system is constructed [8]:

$$
\begin{gathered}
\dot{x}=z+(y-a) x+w, \\
\dot{y}=1-b y-x^{2}, \\
\dot{z}=-x-c z, \\
\dot{w}=-d x y-k w,
\end{gathered}
$$


where $a, b, c, d$, and $k$ are the parameters of the system, and they are positive numbers. When $a=0.9, b=0.2, c=1.5, d=$ 0.2 , and $k=0.17$, system (2) presents hyperchaotic behavior [8]. When the initial value of system (2) is taken as $(1,3,2,5)$ and $t \in(0,1000)$, the phase portraits of system (2) are shown in Figures 1(a)-1(d).

Chaos of financial systems is mostly hyperchaos. In order to achieve financial synchronous development of developed and developing countries or different areas, we need to solve more problems of synchronization of hyperchaotic financial systems.

The projective synchronization discussed in this paper is defined as two relative chaotic dynamical systems can be synchronous with a desired scaling factor [2].

To discuss projective synchronization of the hyperchaotic financial system (2), we rewrite the system (2) as follows:

$$
\begin{aligned}
\left(\begin{array}{c}
\dot{x} \\
\dot{y} \\
\dot{z} \\
\dot{w}
\end{array}\right)= & \left(\begin{array}{cccc}
-a & 0 & 1 & 1 \\
0 & -b & 0 & 0 \\
-1 & 0 & -c & 0 \\
0 & 0 & 0 & -k
\end{array}\right)\left(\begin{array}{c}
x \\
y \\
z \\
w
\end{array}\right) \\
& +\left(\begin{array}{l}
0 \\
1 \\
0 \\
0
\end{array}\right)+\left(\begin{array}{c}
x y \\
-x^{2} \\
0 \\
-d x y
\end{array}\right) .
\end{aligned}
$$

In order to facilitate design of controller, using the thought of state transition, we construct the following response system for the given system (3):

$$
\begin{aligned}
\left(\begin{array}{c}
\dot{x}_{s} \\
\dot{y}_{s} \\
\dot{z}_{s} \\
\dot{w}_{s}
\end{array}\right)= & \left(\begin{array}{cccc}
-a & 0 & 1 & 1 \\
0 & -b & 0 & 0 \\
-1 & 0 & -c & 0 \\
0 & 0 & 0 & -k
\end{array}\right)\left(\begin{array}{l}
x_{s} \\
y_{s} \\
z_{s} \\
w_{s}
\end{array}\right) \\
& +\alpha\left[\left(\begin{array}{l}
0 \\
1 \\
0 \\
0
\end{array}\right)+\left(\begin{array}{c}
x y \\
-x^{2} \\
0 \\
-d x y
\end{array}\right)\right]+\mathbf{u},
\end{aligned}
$$

where $\alpha$ is a scaling factor and $\mathbf{u}$ is the external input control vector.

Denote $\mathbf{x}=(x, y, z, w)^{\mathrm{T}}, \mathbf{y}=\left(x_{s}, y_{s}, z_{s}, w_{s}\right)^{\mathrm{T}}$, and $\mathbf{e}=$ $\left(e_{1}, e_{2}, e_{3}, e_{4}\right)^{\mathrm{T}}$. If we define the error system as $\mathbf{e}=\mathbf{y}-\alpha \mathbf{x}$, we can get the error system

$$
\dot{\mathbf{e}}=A \mathbf{e}+\mathbf{u},
$$

where

$$
A=\left(\begin{array}{cccc}
-a & 0 & 1 & 1 \\
0 & -b & 0 & 0 \\
-1 & 0 & -c & 0 \\
0 & 0 & 0 & -k
\end{array}\right)
$$

Theorem 1. If we design the controller $\mathbf{u}=B \mathbf{e}$ for the error system (5), then the system (5) is asymptotically stable at the origin, where

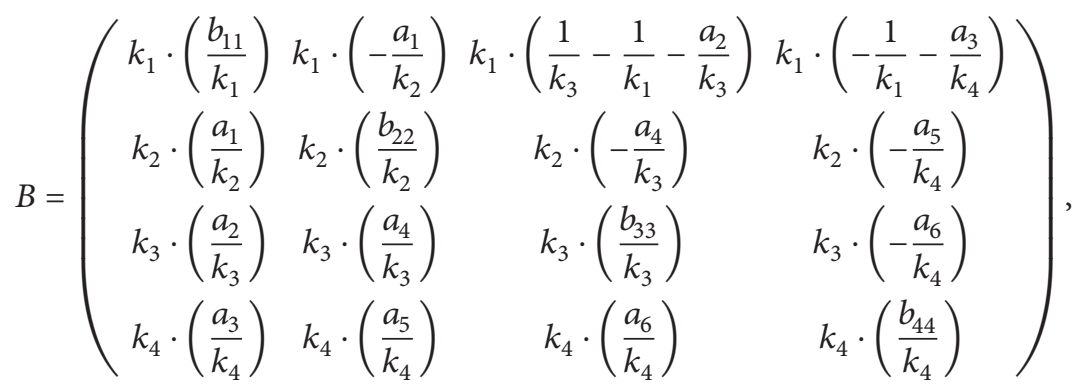

$$
\begin{aligned}
& b_{11} \leq a, \quad b_{22} \leq b, \quad b_{33} \leq c, \quad b_{44} \leq k ; \\
& a_{i} \in R(i=1,2,3,4,5,6), \quad k_{i} \in R^{+}(i=1,2,3,4) .
\end{aligned}
$$

In order to prove Theorem 1, we introduce Lemma 2 firstly.

Lemma 2. Suppose a dynamic system can be written as

$$
\left(\begin{array}{c}
\dot{x}_{1} \\
\dot{x}_{2} \\
\vdots \\
\dot{x}_{n}
\end{array}\right)=\left(\begin{array}{cccc}
k_{1} a_{11} & k_{1} a_{12} & \cdots & k_{1} a_{1 n} \\
k_{2} a_{21} & k_{2} a_{22} & \cdots & k_{2} a_{2 n} \\
\vdots & \vdots & \vdots & \vdots \\
k_{n} a_{n 1} & k_{n} a_{n 2} & \cdots & k_{n} a_{n n}
\end{array}\right)\left(\begin{array}{c}
x_{1} \\
x_{2} \\
\vdots \\
x_{n}
\end{array}\right) .
$$

If the system (8) satisfies the following conditions [9]:

(1) $\forall a_{i j} \in R$,

(2) $a_{i j}=-a_{j i}(i \neq j)$,

(3) $a_{i i} \leq 0$ (not all $a_{i i}$ are equal to zero),

(4) $\forall k_{i}>0$,

then the states of system (8) will decrease to zero gradually. 


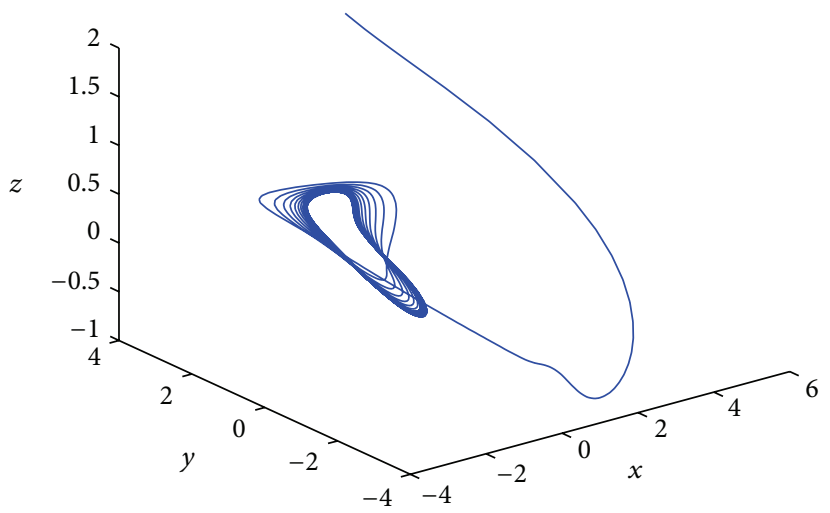

(a) $3 \mathrm{D}$ view in the $x-y-z$ space

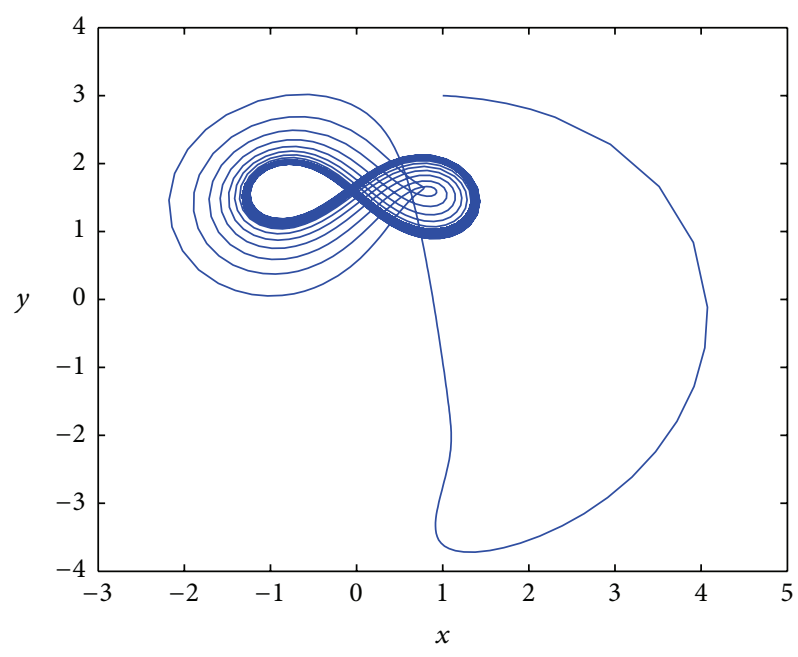

(c) Projection onto the $x-y$ plane

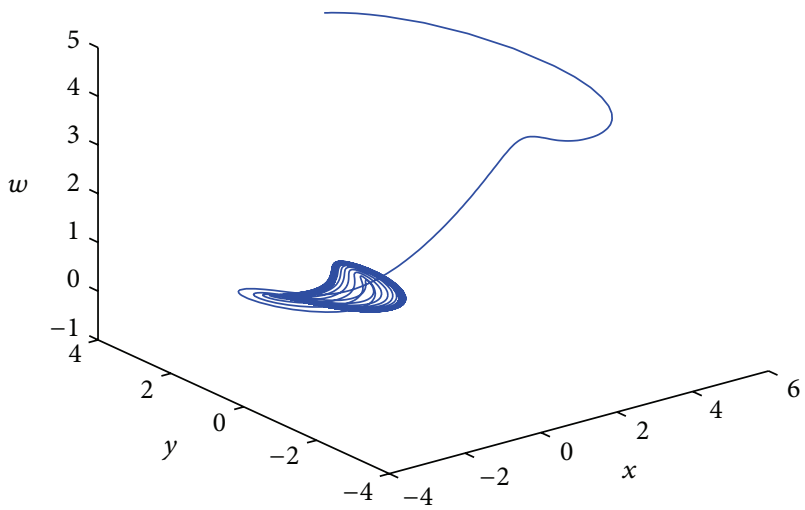

(b) $3 \mathrm{D}$ view in the $x-y-w$ space

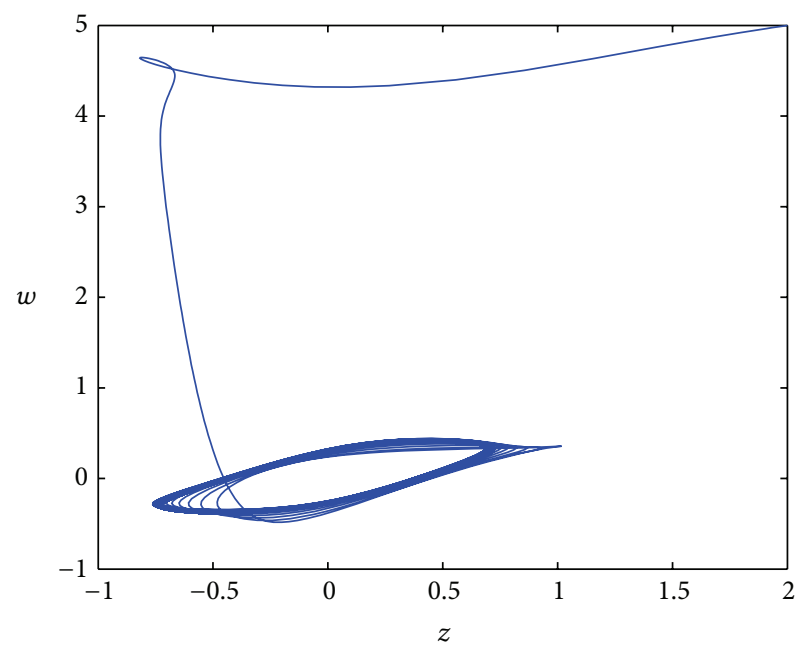

(d) Projection onto the $z-w$ plane

FIgURE 1: Phase portraits of hyperchaotic finance system (2).

Prove Theorem 1 as follows.

Proof. Let controller be $\mathbf{u}=B \mathbf{e}$, where $B=\left(b_{i j}\right)_{4 \times 4}$ is a $4 \times 4$ order constant matrix to be designed; $\mathbf{e}=\left(e_{1}, e_{2}, e_{3}, e_{4}\right)^{\mathrm{T}}$. We rewrite the error system (5) as

$$
\dot{\mathbf{e}}=(c+B) \mathbf{e},
$$

where

$$
\begin{aligned}
C & =\left(c_{i j}\right)_{4 \times 4} \\
& =\left(\begin{array}{cccc}
k_{1} \cdot\left(-\frac{a}{k_{1}}\right) & 0 & k_{1} \cdot\left(\frac{1}{k_{1}}\right) & k_{1} \cdot\left(\frac{1}{k_{1}}\right) \\
0 & k_{2} \cdot\left(-\frac{b}{k_{2}}\right) & 0 & 0 \\
k_{3} \cdot\left(-\frac{1}{k_{3}}\right) & 0 & k_{3} \cdot\left(-\frac{c}{k_{3}}\right) & 0 \\
0 & 0 & 0 & k_{4} \cdot\left(-\frac{k}{k_{4}}\right)
\end{array}\right) .
\end{aligned}
$$

In order to make the system (9) satisfy Lemma 2, design matrix $B$ to satisfy conditions (11) and (12) as follows:

$$
-\frac{a}{k_{1}}+\frac{b_{11}}{k_{1}} \leq 0
$$

$$
\begin{aligned}
& -\frac{b}{k_{2}}+\frac{b_{22}}{k_{2}} \leq 0, \\
& -\frac{c}{k_{3}}+\frac{b_{33}}{k_{3}} \leq 0, \\
& -\frac{k}{k_{4}}+\frac{b_{44}}{k_{4}} \leq 0,
\end{aligned}
$$

$$
\begin{gathered}
\frac{b_{12}}{k_{1}}=-\frac{b_{21}}{k_{2}}, \\
\frac{1}{k_{1}}+\frac{b_{13}}{k_{1}}=-\left(-\frac{1}{k_{3}}+\frac{b_{31}}{k_{3}}\right), \\
\frac{1}{k_{1}}+\frac{b_{14}}{k_{1}}=-\frac{b_{14}}{k_{4}}, \\
\frac{b_{23}}{k_{2}}=-\frac{b_{32}}{k_{3}},
\end{gathered}
$$




$$
\begin{aligned}
& \frac{b_{24}}{k_{2}}=-\frac{b_{42}}{k_{4}}, \\
& \frac{b_{34}}{k_{3}}=-\frac{b_{43}}{k_{4}} .
\end{aligned}
$$

It can be seen from (11) that $b_{11} \leq a, b_{22} \leq b, b_{33} \leq c$, and $b_{44} \leq k$.

Let the coefficient matrix of (12) be $H$, and let its augmented matrix be $\bar{H}$. After calculation, we can get $r(H)=$ $r(\bar{H})$, and the numbers of unknowns are greater than the numbers of (12), so (12) has infinitely many solutions. Note that all solutions of (12) are as follows:

$$
\begin{aligned}
\eta= & \left(b_{12}, b_{21}, b_{13}, b_{31}, b_{14}, b_{41}, b_{23}, b_{32}, b_{24}, b_{42}, b_{34}, b_{43}\right) \\
= & \left(-\frac{a_{1} k_{1}}{k_{2}}, a_{1}, \frac{k_{1}}{k_{3}}-1-\frac{a_{2} k_{1}}{k_{3}}, a_{2},-1-\frac{a_{3} k_{1}}{k_{4}}, a_{3},\right. \\
& \left.-\frac{a_{4} k_{2}}{k_{3}}, a_{4},-\frac{a_{5} k_{2}}{k_{4}}, a_{5},-\frac{a_{6} k_{3}}{k_{4}}, a_{6}\right)
\end{aligned}
$$

where $a_{i} \in R(i=1,2,3,4,5,6) ; k_{i} \in R^{+}(i=1,2,3,4)$.

Therefore integrate (11) and (12) to obtain the matrix $B$ :

$$
B=\left(\begin{array}{cccc}
k_{1} \cdot\left(\frac{b_{11}}{k_{1}}\right) & k_{1} \cdot\left(-\frac{a_{1}}{k_{2}}\right) & k_{1} \cdot\left(\frac{1}{k_{3}}-\frac{1}{k_{1}}-\frac{a_{2}}{k_{3}}\right) & k_{1} \cdot\left(-\frac{1}{k_{1}}-\frac{a_{3}}{k_{4}}\right) \\
k_{2} \cdot\left(\frac{a_{1}}{k_{2}}\right) & k_{2} \cdot\left(\frac{b_{22}}{k_{2}}\right) & k_{2} \cdot\left(-\frac{a_{4}}{k_{3}}\right) & k_{2} \cdot\left(-\frac{a_{5}}{k_{4}}\right) \\
k_{3} \cdot\left(\frac{a_{2}}{k_{3}}\right) & k_{3} \cdot\left(\frac{a_{4}}{k_{3}}\right) & k_{3} \cdot\left(\frac{b_{33}}{k_{3}}\right) & k_{3} \cdot\left(-\frac{a_{6}}{k_{4}}\right) \\
k_{4} \cdot\left(\frac{a_{3}}{k_{4}}\right) & k_{4} \cdot\left(\frac{a_{5}}{k_{4}}\right) & k_{4} \cdot\left(\frac{a_{6}}{k_{4}}\right) & k_{4} \cdot\left(\frac{b_{44}}{k_{4}}\right)
\end{array}\right) .
$$

So system (9) becomes

$$
\left(\begin{array}{c}
\dot{e}_{1} \\
\dot{e}_{2} \\
\dot{e}_{3} \\
\dot{e}_{4}
\end{array}\right)=\left(\begin{array}{cccc}
k_{1} \cdot\left(-\frac{a}{k_{1}}+\frac{b_{11}}{k_{1}}\right) & k_{1} \cdot\left(-\frac{a_{1}}{k_{2}}\right) & k_{1} \cdot\left(\frac{1}{k_{3}}-\frac{a_{2}}{k_{3}}\right) & k_{1} \cdot\left(-\frac{a_{3}}{k_{4}}\right) \\
k_{2} \cdot\left(\frac{a_{1}}{k_{2}}\right) & k_{2} \cdot\left(-\frac{b}{k_{2}}+\frac{b_{22}}{k_{2}}\right) & k_{2} \cdot\left(-\frac{a_{4}}{k_{3}}\right) & k_{2} \cdot\left(-\frac{a_{5}}{k_{4}}\right) \\
k_{3} \cdot\left(-\frac{1}{k_{3}}+\frac{a_{2}}{k_{3}}\right) & k_{3} \cdot\left(\frac{a_{4}}{k_{3}}\right) & k_{3} \cdot\left(-\frac{c}{k_{3}}+\frac{b_{33}}{k_{3}}\right) & k_{3} \cdot\left(-\frac{a_{6}}{k_{4}}\right) \\
k_{4} \cdot\left(\frac{a_{3}}{k_{4}}\right) & k_{4} \cdot\left(\frac{a_{5}}{k_{4}}\right) & k_{4} \cdot\left(\frac{a_{6}}{k_{4}}\right) & k_{4} \cdot\left(-\frac{k}{k_{4}}+\frac{b_{44}}{k_{4}}\right)
\end{array}\right)\left(\begin{array}{l}
e_{1} \\
e_{2} \\
e_{3} \\
e_{4}
\end{array}\right) .
$$

According to Lemma 2, the system (15) is asymptotically stable at the origin; namely, the system (5) is asymptotically stable at the origin.

Remark 3. The error system (5) is asymptotically stable at the origin; namely, the system (3) and system (4) achieve the projective synchronization.

We take $b_{11}=0.5, b_{22}=0.1, b_{33}=1$, and $b_{44}=0.1$; $a_{1}=1, a_{2}=2, a_{3}=3, a_{4}=4, a_{5}=5$, and $a_{6}=6 ; k_{1}=$ $1, k_{2}=2, k_{3}=3$, and $k_{4}=4$. Then if we take $a=0.9$, $b=0.2, c=1.5, d=0.2, k=0.17$, and $\alpha=1 / 2$; the initial values of drive system (3) and response system (4) are taken as $(1,2,0.5,0.5)$ and $(1,3,2,5)$, respectively. So the initial value of error system $(5)$ is $(0,1,1.5,4.5)$, and the state variables of system (5) varying with time $t$ are shown in Figures 2(a)-2(e).
Remark 4. To make the structure of the controller as simple as possible, we take $b_{i i}=0, i=1,2,3,4, a_{i}=0, i=1,2, \ldots, 6$, and $k_{i}=1, i=1,2,3,4$. So we can get control matrix

$$
\begin{gathered}
B=\left(\begin{array}{cccc}
0 & 0 & k_{1} \cdot\left(\frac{1}{k_{3}}-\frac{1}{k_{1}}\right) & k_{1} \cdot\left(-\frac{1}{k_{1}}\right) \\
0 & 0 & 0 & 0 \\
0 & 0 & 0 & 0 \\
0 & 0 & 0 & 0
\end{array}\right), \\
k \\
k_{i} \in R^{+}, \quad i=1,2,3,4
\end{gathered}
$$

which is a special case.

Remark 5. When $\alpha=1$, the system (3) and system (4) are of complete synchronization [10]; when $\alpha=-1$, the system (3) and system (4) are antisynchronization [11]. 

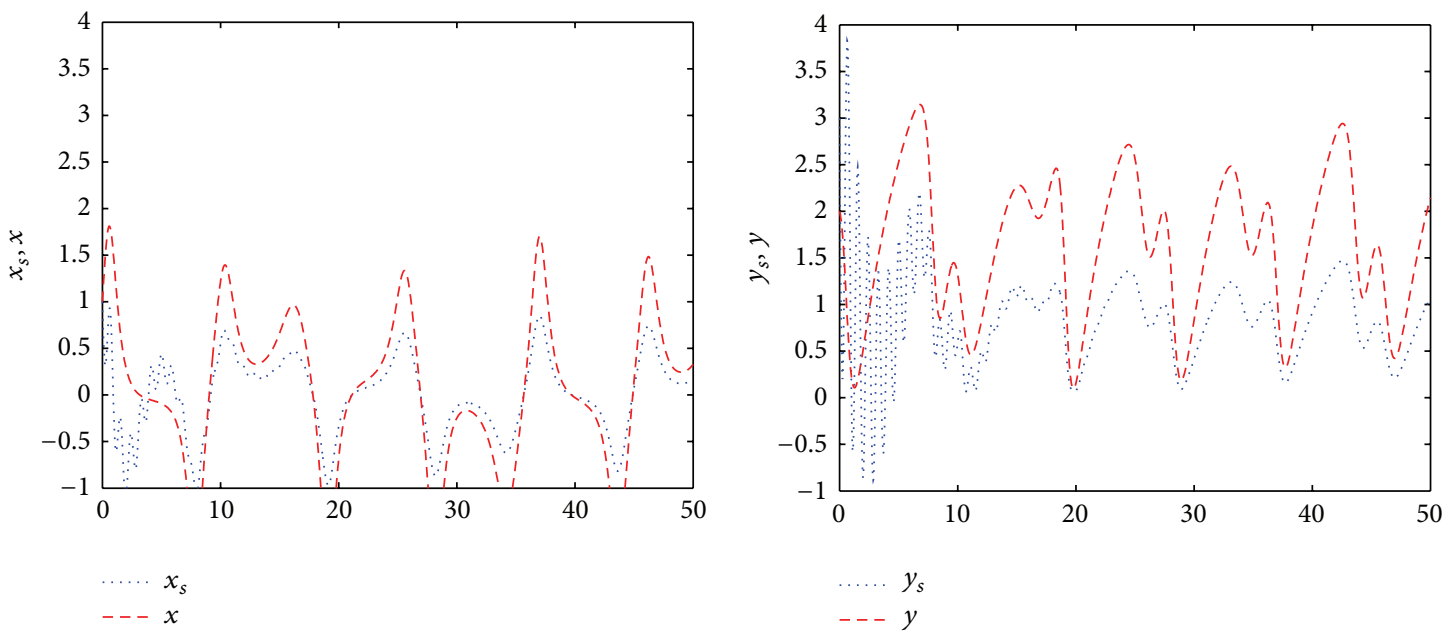

(a) Time evolutions of $x_{s}$ and $x$

(b) Time evolutions of $y_{s}$ and $y$
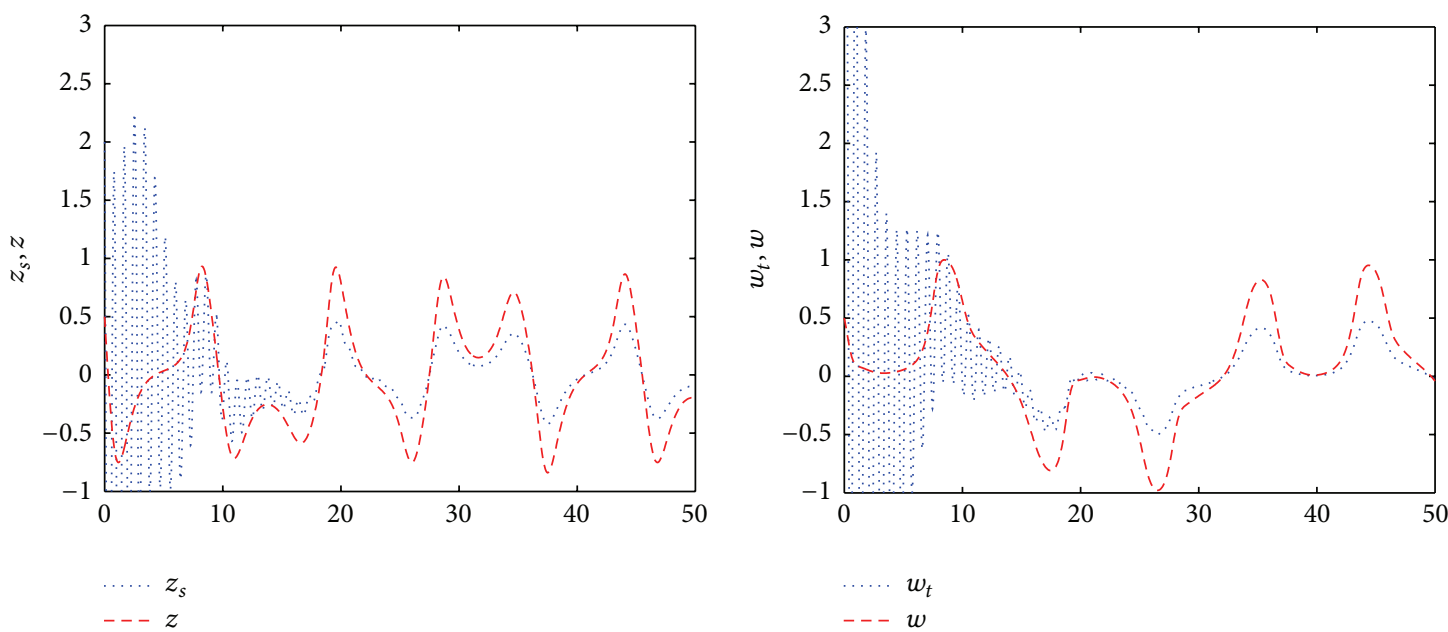

(c) Time evolutions of $z_{s}$ and $z$

(d) Time evolutions of $w_{s}$ and $w$

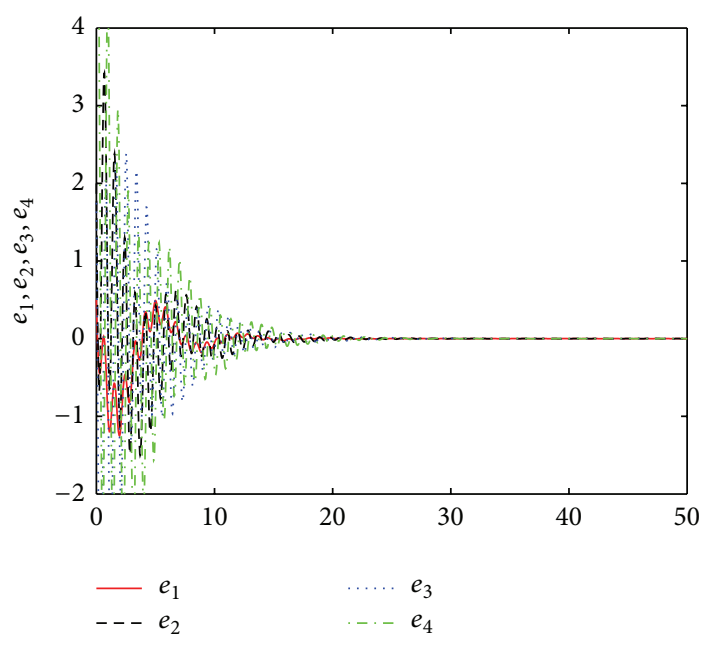

(e) Time evolutions of $e_{1}, e_{2}, e_{3}$, and $e_{4}$

FIGURE 2: Time evolutions of the state variables of system (5). 


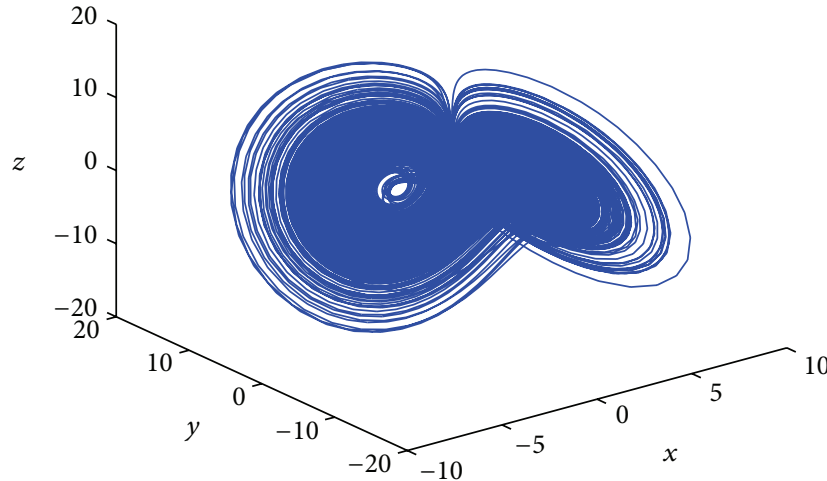

(a) $3 \mathrm{D}$ view in the $x-y-z$ space

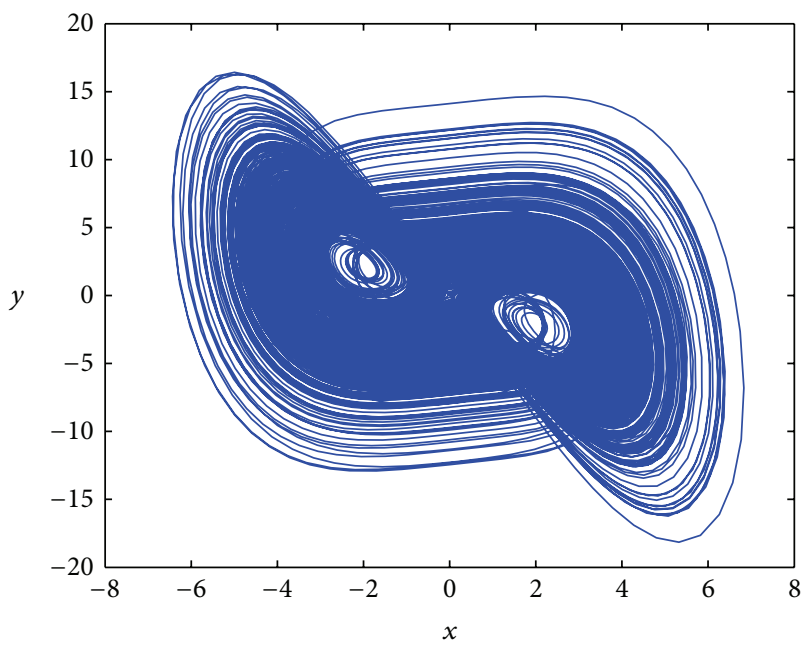

(c) Projection onto the $x-y$ plane

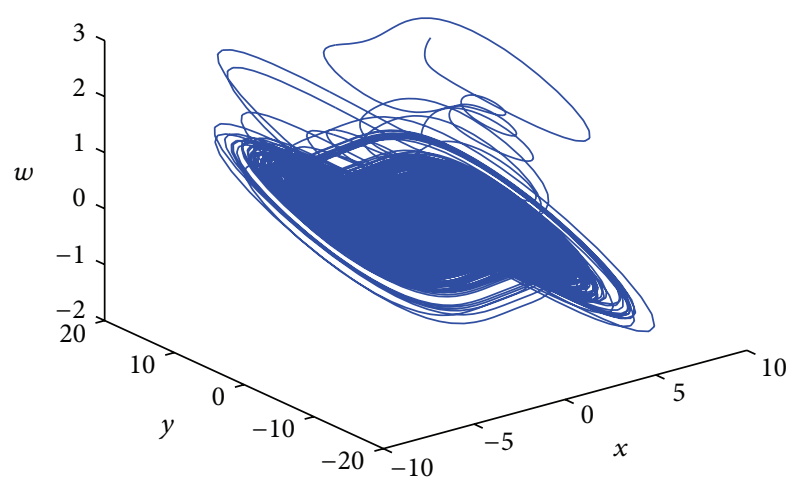

(b) $3 \mathrm{D}$ view in the $x-y-w$ space

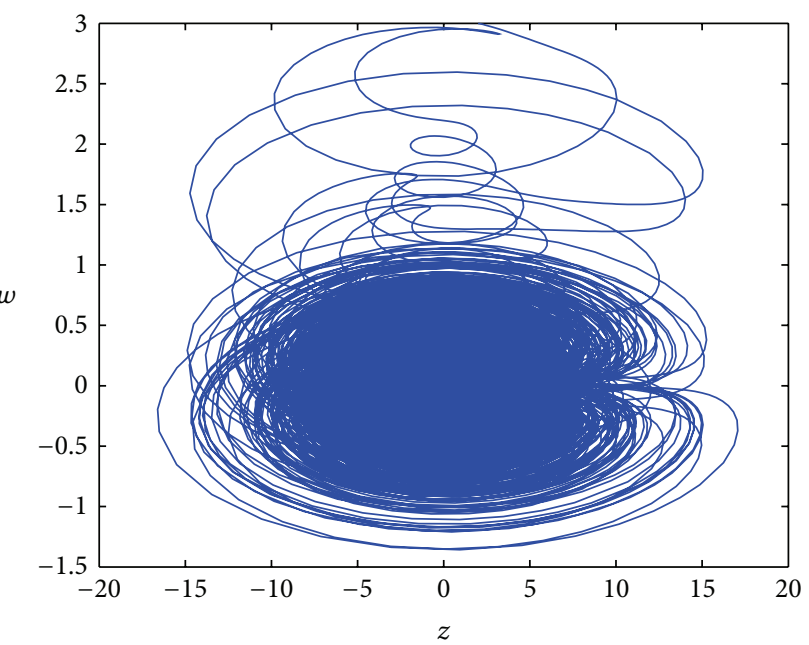

(d) Projection onto the $z-w$ plane

Figure 3: Phase portraits of hyperchaotic finance system (17).

Remark 6. When $k_{1}=k_{2}=k_{3}=k_{4}=1$, the coefficient matrix of system (15) is the antisymmetric matrix in [12]. That is to say, they have the same control scheme.

\section{Projective Synchronization of Two Different Hyperchaotic Financial Systems}

In the real world, we will not only meet synchronization of two identical hyperchaotic financial systems, but also encounter more often the one of two different hyperchaotic financial systems. Here we consider the projective synchronization of the system (2) and the following system (17).

Ding et al. [13] introduced a state feedback controller $w_{t}$ and constructed a new hyperchaotic financial system:

$$
\begin{gathered}
\dot{x}_{t}=-a^{\prime}\left(x_{t}+y_{t}\right)+w_{t}, \\
y_{t}^{\prime}=-y_{t}-a^{\prime} x_{t} z_{t}, \\
\dot{z}_{t}=b^{\prime}+a^{\prime} x_{t} y_{t}, \\
\dot{w}_{t}=-c^{\prime} x_{t} z_{t}-d^{\prime} w_{t},
\end{gathered}
$$

where $a^{\prime}$ and $b^{\prime}$ are parameters of the system (17), $c^{\prime}$ is a constant $\left(c^{\prime}=0.2\right)$, and $d^{\prime}$ is a control parameter. And when $a^{\prime}=3, b^{\prime}=15, c^{\prime}=0.2$, and $d^{\prime}=0.12$, system (17) presents hyperchaotic behavior [13]; the initial value of system (17) is taken as $(1.5,3.2,2,3)$; take $t \in(0,1000)$; the phase portraits of system (17) are shown in Figures 3(a)-3(d).

Let the system (3) be the drive system, and let the system (17) be response system. So the system (17) becomes

$$
\begin{aligned}
\left(\begin{array}{c}
\dot{x}_{t} \\
\dot{y}_{t} \\
\dot{z}_{t} \\
\dot{w}_{t}
\end{array}\right)= & \left(\begin{array}{cccc}
-a^{\prime} & -a^{\prime} & 0 & 1 \\
0 & -1 & 0 & 0 \\
0 & 0 & 0 & 0 \\
0 & 0 & 0 & -d^{\prime}
\end{array}\right)\left(\begin{array}{c}
x_{t} \\
y_{t} \\
z_{t} \\
w_{t}
\end{array}\right) \\
& +\left(\begin{array}{c}
0 \\
-a^{\prime} x_{t} z_{t} \\
a^{\prime} x_{t} y_{t} \\
-c^{\prime} x_{t} z_{t}
\end{array}\right)+\left(\begin{array}{c}
0 \\
0 \\
b^{\prime} \\
0
\end{array}\right)+\mathbf{u} .
\end{aligned}
$$

We denote

$$
A=\left(\begin{array}{cccc}
-a & 0 & 1 & 1 \\
0 & -b & 0 & 0 \\
-1 & 0 & -c & 0 \\
0 & 0 & 0 & -k
\end{array}\right), \quad \mathbf{h}_{1}=\left(\begin{array}{l}
0 \\
1 \\
0 \\
0
\end{array}\right)
$$




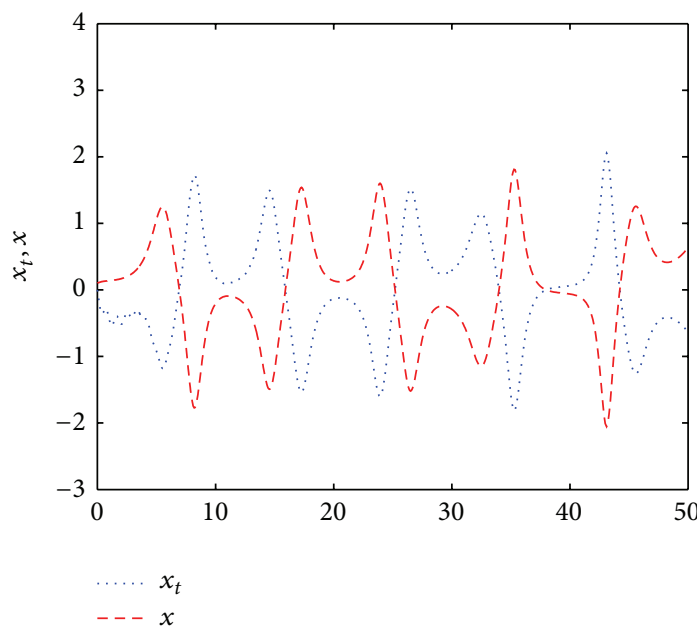

(a) Time evolutions of $x_{t}$ and $x$

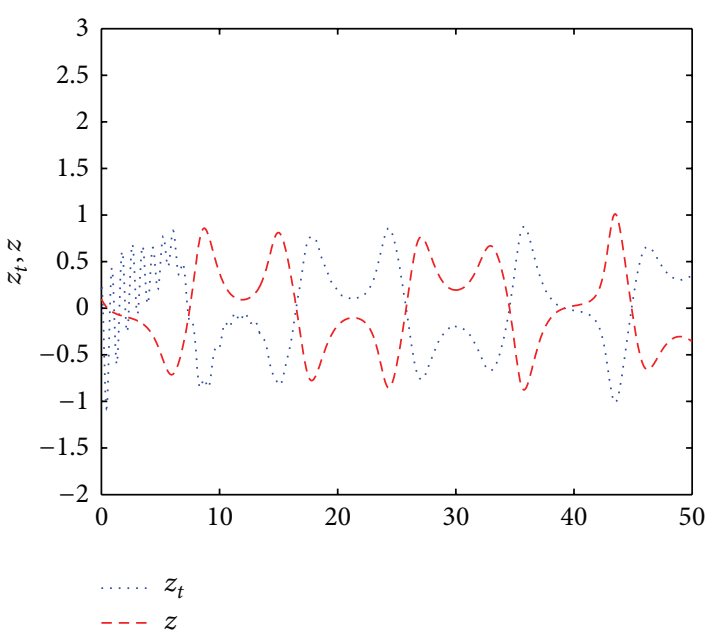

(c) Time evolutions of $z_{t}$ and $z$

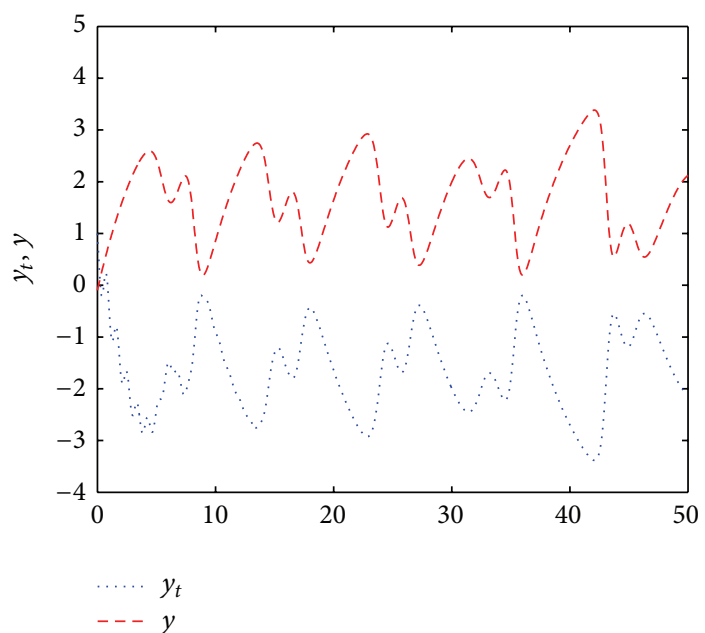

(b) Time evolutions of $y_{t}$ and $y$

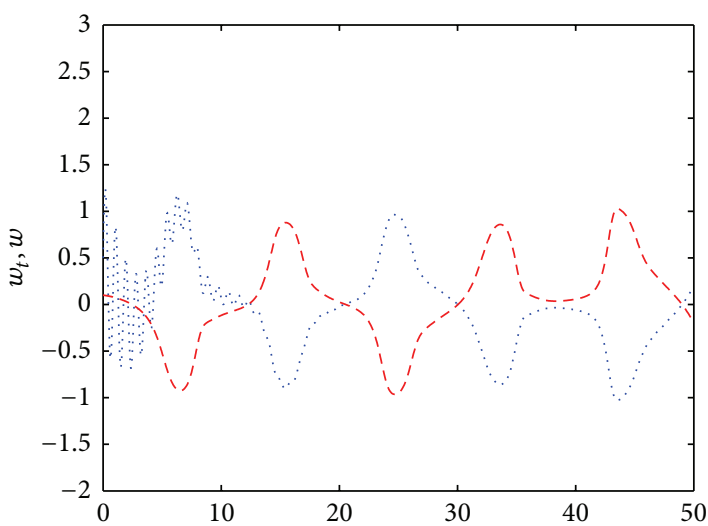

…. $w_{t}$

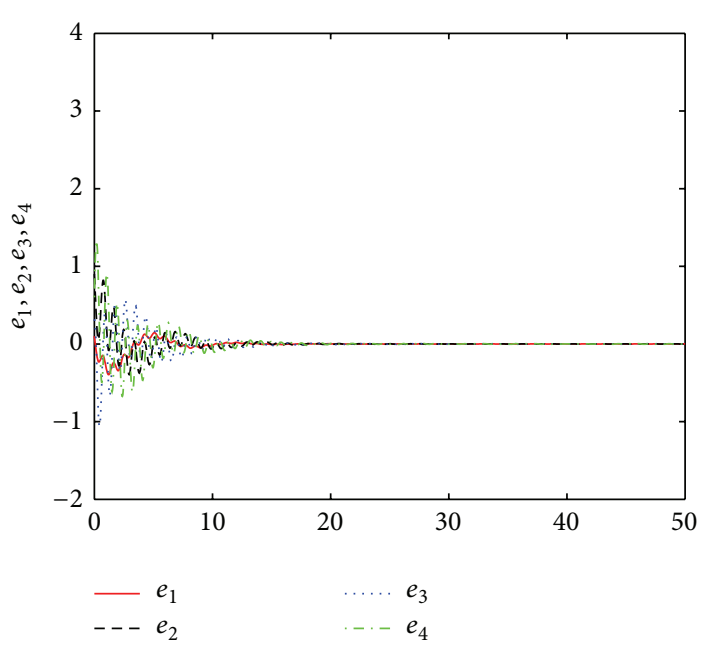

(e) Time evolutions of $e_{1}, e_{2}, e_{3}$, and $e_{4}$

FIgURE 4: Time evolutions of the state variables of system (3) and system (20). 


$$
\begin{gathered}
f(\mathbf{x})=\left(\begin{array}{c}
x y \\
-x^{2} \\
0 \\
-d x y
\end{array}\right), \quad \mathbf{x}=\left(\begin{array}{c}
x \\
y \\
z \\
w
\end{array}\right), \\
D=\left(\begin{array}{cccc}
-a^{\prime} & -a^{\prime} & 0 & 1 \\
0 & -1 & 0 & 0 \\
0 & 0 & 0 & 0 \\
0 & 0 & 0 & -d^{\prime}
\end{array}\right), \quad \mathbf{h}_{2}=\left(\begin{array}{c}
0 \\
0 \\
b^{\prime} \\
0
\end{array}\right), \\
g(\mathbf{y})=\left(\begin{array}{c}
0 \\
-a^{\prime} x_{t} z_{t} \\
a^{\prime} x_{t} y_{t} \\
-c^{\prime} x_{t} z_{t}
\end{array}\right), \quad \mathbf{y}=\left(\begin{array}{c}
x_{t} \\
y_{t} \\
z_{t} \\
w_{t}
\end{array}\right) .
\end{gathered}
$$

Let $\mathbf{u}=(A-D) \mathbf{y}+\alpha\left(\mathbf{h}_{1}+f(\mathbf{x})\right)-\mathbf{h}_{2}-g(\mathbf{y})+E \mathbf{e}$, where $E=\left(e_{i j}\right)_{4 \times 4}$ is a $4 \times 4$ order constant matrix to be designed, and $\mathbf{e}=\left(e_{1}, e_{2}, e_{3}, e_{4}\right)^{\mathrm{T}}$. So the response system (18) becomes

$$
\left(\begin{array}{c}
\dot{x}_{t} \\
\dot{y}_{t} \\
\dot{z}_{t} \\
\dot{w}_{t}
\end{array}\right)=\left(\begin{array}{cccc}
-a & 0 & 1 & 1 \\
0 & -b & 0 & 0 \\
-1 & 0 & -c & 0 \\
0 & 0 & 0 & -k
\end{array}\right)\left(\begin{array}{c}
x_{t} \\
y_{t} \\
z_{t} \\
w_{t}
\end{array}\right)
$$

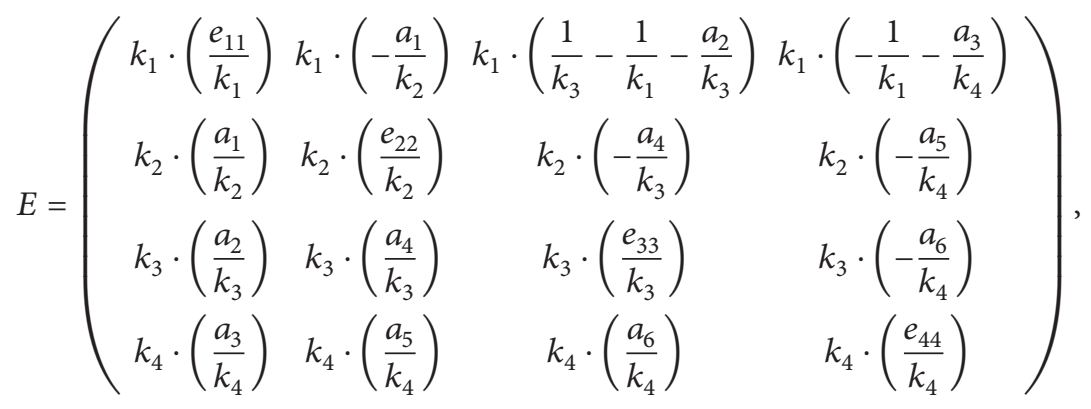

$$
\begin{aligned}
& e_{11} \leq a, \quad e_{22} \leq b, \quad e_{33} \leq c, \quad e_{44} \leq k, \\
& a_{i} \in R, i=1,2, \ldots, 6 ; \quad k_{i} \in R^{+}, i=1,2,3,4 .
\end{aligned}
$$

Then the system (21) is asymptotically stable at the origin.

Proof. If $\mathbf{u}=(A-D) \mathbf{y}+\alpha\left(\mathbf{h}_{1}+f(\mathbf{x})\right)-\mathbf{h}_{2}-g(\mathbf{y})+E \mathbf{e}$, according to the definition of the error system, the error system of the driven system (3) and response system (18) is system (21); namely,

$$
\dot{\mathbf{e}}=(C+E) \mathbf{e} .
$$

System (24) has the same structure with the system (9), so we can get $E=B$; namely,

$$
E=\left(\begin{array}{cccc}
k_{1} \cdot\left(\frac{e_{11}}{k_{1}}\right) & k_{1} \cdot\left(-\frac{a_{1}}{k_{2}}\right) & k_{1} \cdot\left(\frac{1}{k_{3}}-\frac{1}{k_{1}}-\frac{a_{2}}{k_{3}}\right) & k_{1} \cdot\left(-\frac{1}{k_{1}}-\frac{a_{3}}{k_{4}}\right) \\
k_{2} \cdot\left(\frac{a_{1}}{k_{2}}\right) & k_{2} \cdot\left(\frac{e_{22}}{k_{2}}\right) & k_{2} \cdot\left(-\frac{a_{4}}{k_{3}}\right) & k_{2} \cdot\left(-\frac{a_{5}}{k_{4}}\right) \\
k_{3} \cdot\left(\frac{a_{2}}{k_{3}}\right) & k_{3} \cdot\left(\frac{a_{4}}{k_{3}}\right) & k_{3} \cdot\left(\frac{e_{33}}{k_{3}}\right) & k_{3} \cdot\left(-\frac{a_{6}}{k_{4}}\right) \\
k_{4} \cdot\left(\frac{a_{3}}{k_{4}}\right) & k_{4} \cdot\left(\frac{a_{5}}{k_{4}}\right) & k_{4} \cdot\left(\frac{a_{6}}{k_{4}}\right) & k_{4} \cdot\left(\frac{e_{44}}{k_{4}}\right)
\end{array}\right) .
$$


Therefore, the system (24) becomes

$$
\dot{\mathbf{e}}=(C+E) \mathbf{e},
$$

where

$$
C+E=\left(\begin{array}{cccc}
k_{1} \cdot\left(-\frac{a}{k_{1}}+\frac{e_{11}}{k_{1}}\right) & k_{1} \cdot\left(-\frac{a_{1}}{k_{2}}\right) & k_{1} \cdot\left(\frac{1}{k_{3}}-\frac{a_{2}}{k_{3}}\right) & k_{1} \cdot\left(-\frac{a_{3}}{k_{4}}\right) \\
k_{2} \cdot\left(\frac{a_{1}}{k_{2}}\right) & k_{2} \cdot\left(-\frac{b}{k_{2}}+\frac{e_{22}}{k_{2}}\right) & k_{2} \cdot\left(-\frac{a_{4}}{k_{3}}\right) & k_{2} \cdot\left(-\frac{a_{5}}{k_{4}}\right) \\
k_{3} \cdot\left(-\frac{1}{k_{3}}+\frac{a_{2}}{k_{3}}\right) & k_{3} \cdot\left(\frac{a_{4}}{k_{3}}\right) & k_{3} \cdot\left(-\frac{c}{k_{3}}+\frac{e_{33}}{k_{3}}\right) & k_{3} \cdot\left(-\frac{a_{6}}{k_{4}}\right) \\
k_{4} \cdot\left(\frac{a_{3}}{k_{4}}\right) & k_{4} \cdot\left(\frac{a_{5}}{k_{4}}\right) & k_{4} \cdot\left(\frac{a_{6}}{k_{4}}\right) & k_{4} \cdot\left(-\frac{k}{k_{4}}+\frac{e_{44}}{k_{4}}\right)
\end{array}\right) .
$$

According to Lemma 2, the system (26) tends to zero gradually; namely, the system (21) is driven to the origin gradually.

Remark 8. The system (21) tended to zero gradually; that is, the drive system (3) and response system (18) achieve the projective synchronization.

Generally, we take $e_{11}=0.5, e_{22}=0.1, e_{33}=1$, and $e_{44}=$ $0.1 ; a_{1}=1, a_{2}=2, a_{3}=3, a_{4}=4, a_{5}=5$, and $a_{6}=6$; $k_{1}=1, k_{2}=2, k_{3}=3$, and $k_{4}=4$. And we take $a=0.9$, $b=0.2, c=1.5, d=0.2, k=0.17$, and $\alpha=-1$; the initial values of drive system (3) and response system (17) are taken as $(0.1,-0.1,0.1,0.1)$ and $(0,1,0.2,0.5)$, respectively. So the initial value of error system (21) is $(-0.1,1.1,0.1,0.4)$, and the state variables of system (21) varying with time $t$ are shown in Figures 4(a)-4(e).

\section{Conclusions}

Based on a special matrix structure and the conversion of the state variable, we design the controllers to achieve the projective synchronization of hyperchaotic financial systems in this paper. And the control law is also suitable for synchronous control of other chaotic systems. Theoretical analysis and simulation results show the effectiveness of the method.

\section{Conflict of Interests}

The authors declare that they have no conflict of interests regarding the publication of this paper.

\section{Acknowledgment}

The authors gratefully acknowledge the Scientific and Technological Research Program of Chongqing Municipal Education Commission (Grant no. KJ1400428).

\section{References}

[1] R. Mainieri and J. Rehacek, "Projective synchronization in three-dimensional chaotic systems," Physical Review Letters, vol. 82, no. 15, pp. 3042-3045, 1999.
[2] B. Xin and T. Chen, "Projective synchronization of $N$ dimensional chaotic fractional-order systems via linear state error feedback control," Discrete Dynamics in Nature and Society, vol. 2012, Article ID 191063, 10 pages, 2012.

[3] M. Yang, B. Cai, and G. Cai, "Projective synchronization of a modified three-dimensional chaotic finance system," International Journal of Nonlinear Science, vol. 10, no. 1, pp. 32-38, 2010.

[4] M. Yang, Synchronization analysis for a kind of modified chaotic finance systems [M.S. thesis], Jiangsu University, Jiangsu, China, 2010 (Chinese).

[5] L. Yao and G. Cai, "Chaos synchronization of a new hyperchaotic finance system via a novel chatter free sliding mode control strategy," International Journal of Nonlinear Science, vol. 17, no. 2, pp. 176-181, 2014.

[6] X. Chai, Z. Gan, and C. Shi, "Impulsive synchronization and adaptive-impulsive synchronization of a novel financial hyperchaotic system," Mathematical Problems in Engineering, vol. 2013, Article ID 751616, 10 pages, 2013.

[7] D. Huang and H. Li, The Theory and Method of Nonlinear Economy, Sichuan University Press, Chengdu, China, 1993, (Chinese).

[8] H. Yu, G. Cai, and Y. Li, "Dynamic analysis and control of a new hyperchaotic finance system," Nonlinear Dynamics, vol. 67, no. 3, pp. 2171-2182, 2012.

[9] J. Hu, Y. Han, and L. Zhao, "Synchronizing chaotic systems using control based on a special matrix structure and extending to fractional chaotic systems," Communications in Nonlinear Science and Numerical Simulation, vol. 15, no. 1, pp. 115-123, 2010.

[10] G. M. Mahmoud and E. E. Mahmoud, "Complete synchronization of chaotic complex nonlinear systems with uncertain parameters," Nonlinear Dynamics, vol. 62, no. 4, pp. 875-882, 2010.

[11] X. R. Shi and Z. L. Wang, "Adaptive added-order antisynchronization of chaotic systems with fully unknown parameters," Applied Mathematics and Computation, vol. 215, no. 5, pp. 1711-1717, 2009.

[12] X. Chen and J. Qiu, "Synchronization of N different chaotic systems based on antisymmetric structure," Mathematical Problems in Engineering, vol. 2013, Article ID 742765, 6 pages, 2013.

[13] J. Ding, W. Yang, and H. Yao, "A new modified hyperchaotic finance system and its control," International Journal of Nonlinear Science, vol. 8, no. 1, pp. 59-66, 2009. 


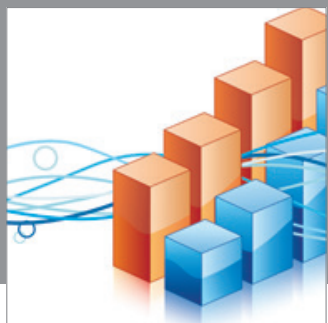

Advances in

Operations Research

mansans

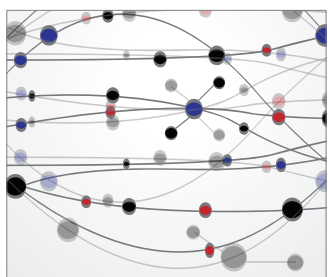

The Scientific World Journal
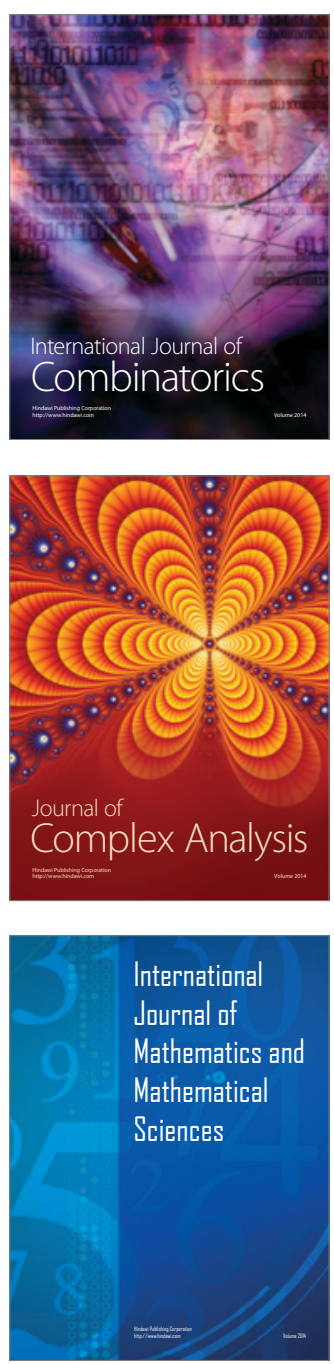
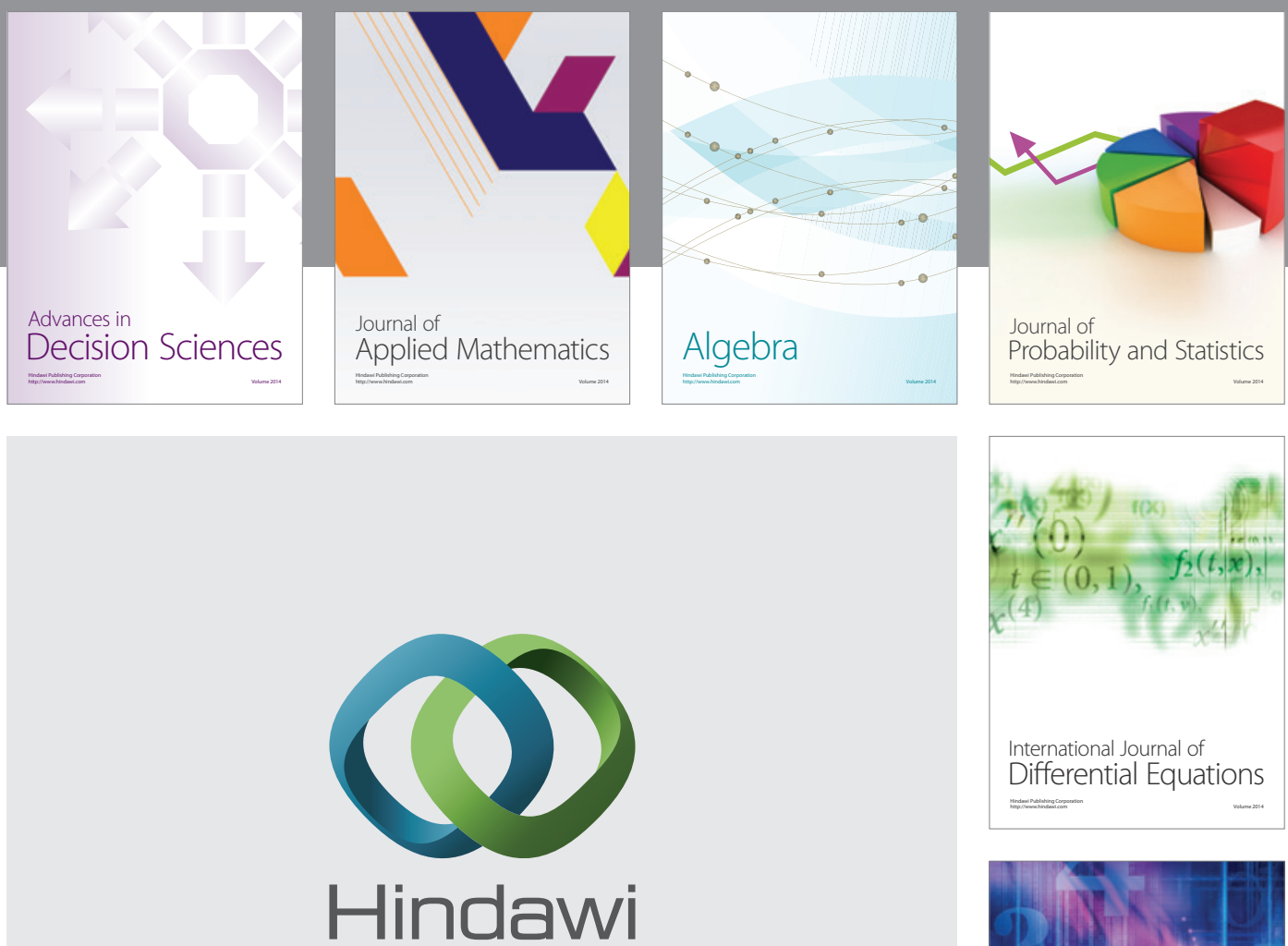

Submit your manuscripts at http://www.hindawi.com
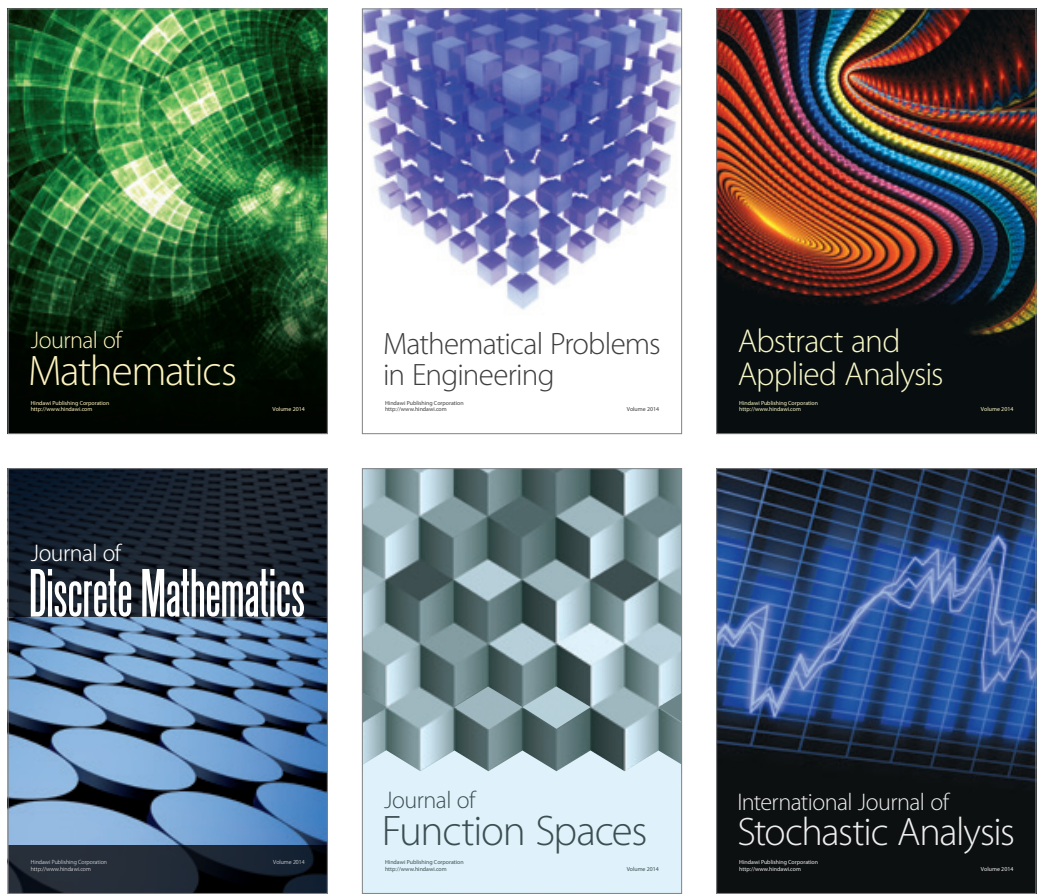

Journal of

Function Spaces

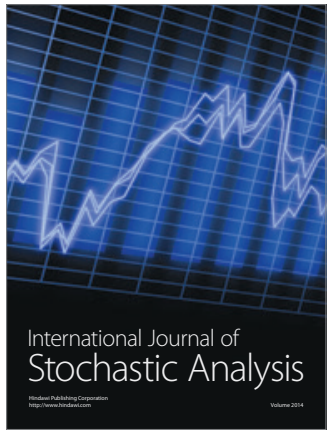

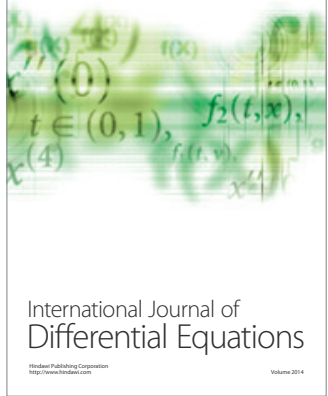
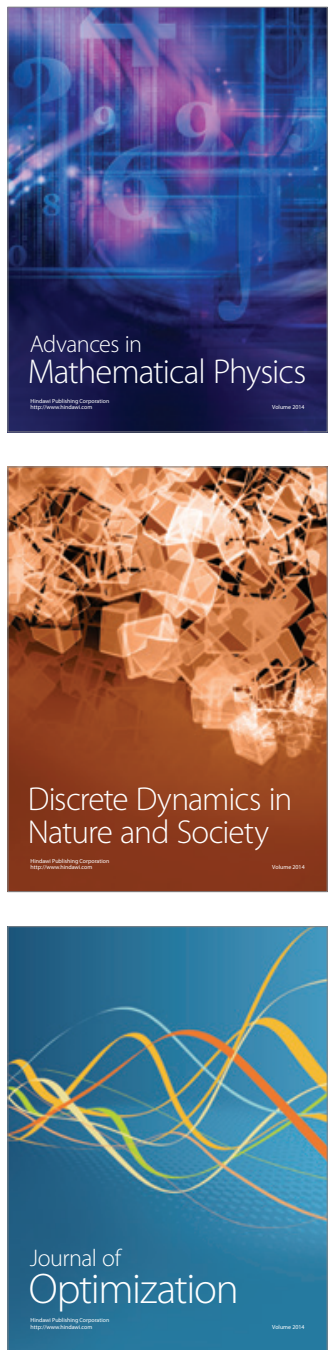